\title{
EVALUATION OF CLINICAL AND PATHOLOGICAL RESPONSE AFTER TWO CYCLES OF NEOADJUVANT CHEMOTHERAPY ON SUDANESE PATIENTS WITH LOCALLY ADVANCED BREAST CANCER
}

\author{
Awad Ali M. Alawad
}

\section{ABSTRACT}

BACKGROUND: The use of neoadjuvant chemotherapy in treating breast cancer has shown efficacy in downstaging primary tumors, and allows breast conservative surgery to be performed instead of mastectomy. This study aims to evaluate patterns of clinical and pathological response after two cycles of neoadjuvant chemotherapy in patients with locally advanced breast cancer.

MATERIALS AND METHODS: This is a prospective study. Ninety-eight patients who presented from April 2009 through May 2011 with locally advanced breast cancer and treated with neoadjuvant chemotherapy were included.

RESULTS: The clinical response rate was $83 \%$; 11 patients $(11.2 \%)$ had a complete clinical remission (cCR); 71 had a partial remission (72.4\%); 13 had stable disease (13.3\%), and 3 had progressive disease (3.1\%). Seven patients had complete pathological response.

CONCLUSION: Neoadjuvant chemotherapy can achieve a high objective response rate in patients with locally advanced breast cancer even after two cycles. We recommend further research to find predictors for response.

KEYWORDS: Breast cancer, Clinical response, Neoadjuvant chemotherapy

\section{DOI: http://dx.doi.org/10.4314/ejhs.v24i1.2}

\section{INTRODUCTION}

The value of adjuvant chemotherapy in treating breast cancer is well documented. The idea of neoadjuvant chemotherapy has been recently applied to breast cancer treatment and studies have shown the efficacy of neoadjuvant chemotherapy in down-staging the primary tumor (1). The use of neoadjuvant chemotherapy thus allows breast conservation surgery to be performed instead of mastectomy. The Milan Group, using a combination of doxorubicin and vincristine, achieved an $80 \%$ response rate with $15 \%$ of patients attaining complete clinical response (2). Tumor shrinkage by the neoadjuvant chemotherapy can be easily monitored clinically both by physicians and patients. For physicians, continuation of treatment is reasonably determined based on efficacy. For patients, compliance with the scheduled courses of chemotherapy is increased because they, themselves, experience the efficacy, which helps them mentally to overcome the unpleasant adverse effects (3). The pathological response to the neoadjuvant chemotherapy provides reliable prognostic information (4).

While locally advanced breast cancer (LABC) is an unusual presentation among women in the western world, in Sudan, this stage is not only common but it also affects women at a younger age (5).

Assistant Professor of Surgery, Faculty of Medicine, University of Medical Sciences and Technology, Sudan Corresponding Author: Awad Ali Mohamed Ahmed Alawad, Email: awadali82@hotmail.com 
This study evaluates the patterns of response to neoadjuvant chemotherapy and its impact on type of surgery in patients with locally advanced breast cancer.

\section{MATERIALS AND METHODS}

A total of ninety-eight patients who presented in April 2009 through May 2011 with locally advanced breast cancer (LABC) were treated with neoadjuvant chemotherapy in the combined breast clinic in National Cancer Institute. In the TNM staging classification, LABC is represented by stage IIIA (T0-N2; T1/2 - N2; T3 - N1/2), stage IIIB (T4, N0-2) and stage IIIC disease (any $\mathrm{T}, \mathrm{N} 3$ ).

Various chemotherapy schedules were used according to standard practice at different times. For much of this period, the standard neoadjuvant treatment regimen was 5-fluorouracil, epirubicin and cyclophosphamide (FEC) with two schedules used: $500 \mathrm{mg} / \mathrm{m}^{2}$ 5-fluorouracil, $75 \mathrm{mg} / \mathrm{m}^{2}$ epirubicin and $500 \mathrm{mg} / \mathrm{m}^{2}$ cyclophosphamide or $600 \mathrm{mg} / \mathrm{m}^{2} 5$-fluorouracil, $60 \mathrm{mg} / \mathrm{m}^{2}$ epirubicin and $600 \mathrm{mg} / \mathrm{m}^{2}$ cyclophosphamide, both for 6 cycles on a 21-day cycle. Forty three patients were treated with the FEC schedule. Other chemotherapy schedules used included single agent docetaxel $\left(100 \mathrm{mg} / \mathrm{m}^{2}\right.$ q 21 for four cycles, 22 patients), AC (adriamycin $60 \mathrm{mg} / \mathrm{m}^{2}$, cyclophosphamide $600 \mathrm{mg} / \mathrm{m}^{2}$ q21 for four cycles, 15 patients), CMF (oral cyclophosphamide $100 \mathrm{mg} / \mathrm{m}^{2}$ day $1-14$ and intravenous methotrexate $40 \mathrm{mg} / \mathrm{m}^{2}$ and 5-fluorouracil 600 $\mathrm{mg} / \mathrm{m}^{2}$ days 1 and 8 , q28 for six cycles, five patients), MMM (methotrexate $30 \mathrm{mg} / \mathrm{m}^{2}$ and mitoxantrone $7 \mathrm{mg} / \mathrm{m}^{2}$ days 1 and 22, mitomycin $\mathrm{C} 7 \mathrm{mg} / \mathrm{m}^{2}$ day $1, \mathrm{q} 42$ for 3 cycles, six patients), MM (methotrexate $30 \mathrm{mg} / \mathrm{m}^{2}$ and mitoxantrone 7 $\mathrm{mg} / \mathrm{m}^{2}, \mathrm{q} 21$ for six cycles, seven patients).

Baseline patient and tumor characteristics recorded included age, tumor stage, nodal stage, tumor grade, estrogen receptor (ER) status and progesterone receptor (PR) status. Clinical response was assessed after two cycles of chemotherapy (at the 6th week from baseline measurements) and at the end of the treatment. Union for International Cancer control (UICC) criteria was used in recording response. A complete clinical response (cCR) was achieved when the original mass became impalpable. A partial response (cPR) represented a $50 \%$ or greater reduction in bi-dimensional tumor measurements.

Progressive disease (cPD) was recorded if bi-dimensional measurements increased by $20 \%$ or more. All others were classified as stable disease (cSD). Pathological response was assessed at definitive surgery on completion of neoadjuvant chemotherapy. A pathological complete response ( $\mathrm{pCR}$ ) was noted when there was no evidence of residual tumor on histological examination of the surgical specimen. Patients with residual ductal carcinoma in-situ (DCIS) and no evidence of residual invasive disease were included in this category. The pCR rate was compared by response category after two cycles of chemotherapy. The rates of mastectomy and breast-conserving surgery were noted for all patients undergoing operation and these rates were compared by response categories. All summary statistics were stated with $95 \%$ confidence limits. An appropriate method for small samples was applied to the percentages and odds ratios were derived using logistic regression methods (SPSS version 17).

The consent of the patients was obtained. A full explanation of the purposes and nature of the study was conveyed to them. The potential participants were clearly assured that their participation in this study was voluntary and that they could withdraw at any stage and that any data obtained would be treated confidentially and would be used for the purpose of the research only.

\section{RESULTS}

Ninety-eight patients receiving primary chemotherapy for locally advanced breast cancer were studied. The median age of the patients at the time of diagnosis was 46 years (range: 2571). About $43.9 \%$ of the patients $(n=43)$ were living in rural areas while $56.1 \%(\mathrm{n}=55)$ came from urban areas. In terms of menopausal status, $61(62.2 \%)$ patients were pre-menopausal while $37(37.8 \%)$ were post-menopausal. The mean tumor diameter measured clinically before neoadjuvant chemotherapy was $9.6 \mathrm{~cm}$ (range 4$18 \mathrm{~cm}$ ). Axillary nodal status was N0 in 3 patients $(3.1 \%), \mathrm{N} 1$ in 22 patients $(22.4 \%), \mathrm{N} 2$ in 66 patients $(67.3 \%)$ and $\mathrm{N} 3$ in 7 patients $(7.1 \%)$. 
Thirteen patients (13.3\%) had Stage IIIa disease, 77 patients $(78.6 \%)$ had Stage IIIb disease and 8 patients $(8.2 \%)$ had Stage IIIc disease.

A histological classification was made: 76 patients $(77.6 \%)$ were classified as invasive ductal carcinoma (IDC), 15 as invasive lobular carcinoma (ILC) (15.3\%) and 7 as other types (7.1\%), including mixed invasive patterns, figure (4). Malignancy grading was also done: $2(2 \%)$ were grade I, $20(20.4 \%)$ were grade II and 76 $(77.6 \%)$ were grade III.

Estrogen receptors showed positivity in 66 patients $(67.3 \%)$, and progesterone receptors in 55 patients $(56.1 \%)$. The mean tumor diameter measured in the surgical sample after neoadjuvant chemotherapy was $2.6 \mathrm{~cm}$ (range 0$12 \mathrm{~cm})$. Eleven patients $(11.2 \%)$ had a clinical complete remission (cCR), 71 had a partial remission (72.4\%), 13 had stable disease (13.3\%) and 3 had progressive disease $(3.1 \%)$. Clinical examination of the axilla revealed a complete response in $16(16.3 \%)$ and an incomplete response in $50(51 \%)$.

Following NAC, 33 patients $(33.7 \%)$ underwent breast conservative surgery (BCS), while 49 (66.3\%) had total mastectomy. All patients had axillary lymph node dissection (ALND).The other 16 patient continued their cycles of chemotherapy. Seven patients $(7.1 \%)$ attained complete pathological response (pCR), 17 (17.3\%) demonstrated partial response, while 58 patients showed pathological stable disease. There was no significant difference in the response rates based on the stage of the disease $(\mathrm{p}=0.036)$.

Comparison between clinical and pathologic response was also made. Based on clinical assessment, 6 of the 11 patients (54.5\%) who were considered as complete clinical responder, in fact, had pCR. On the other hand, one patient of the $71(1.4 \%)$ patients who were considered as partial clinical responders was eventually found to have pCR.

Table 1: Baseline patient and tumour characteristics and the distribution of the characteristics by clinical response after two cycles of chemotherapy, $2011(\mathrm{~N}=98)$.

\begin{tabular}{|llll|}
\hline Parameter & Total population & $\begin{array}{c}\text { Responders after } \\
\text { Two cycles }\end{array}$ & $\begin{array}{c}\text { Non-responders } \\
\text { after two cycles }\end{array}$ \\
\hline $\begin{array}{l}\text { Age } \\
\text { Median }\end{array}$ & 46 & 47 & 50 \\
Tumor stage & & & \\
III a & $13(13.3 \%)$ & $13(15.8 \%)$ & 0 \\
III b & $77(78.6 \%)$ & $67(81.7 \%)$ & $10(62.5 \%)$ \\
III c & $8(8.2 \%)$ & $2(2.5 \%)$ & $6(37.5 \%)$ \\
Nodal stage & $3(3.1 \%)$ & $3(3.6 \%)$ & 0 \\
N0 & $22(22.4 \%)$ & $20(24.4 \%)$ & $2(12.5 \%)$ \\
N1 & $66(67.3 \%)$ & $58(70.7 \%)$ & $8(50 \%)$ \\
N2 & $7(7.1 \%)$ & $1(1.3 \%)$ & $6(37.5 \%)$ \\
N3 & $2(2 \%)$ & $2(2.5 \%)$ & 0 \\
Tumor grade & $20(20.4 \%)$ & $10(12.2 \%)$ & $10(62.5 \%)$ \\
G1 & $76(77.6 \%)$ & $70(85.3 \%)$ & $6(37.5 \%)$ \\
G2 & & & $4(12.5 \%)$ \\
G3 & $66(67.3 \%)$ & $62(75 \%)$ & $14(87.5 \%)$ \\
ER status & $32(32.7 \%)$ & $18(25 \%)$ & \\
+ve & & & \\
-ve & & &
\end{tabular}

Table 2 shows univariate analysis for prediction of a pCR by response at two cycles and for various known prognostic factors, including age, baseline tumour stage, nodal status, histological grade and ER status. Response at two cycles and baseline ER status significantly predict for pCR in the univariate model. 
Table 2: Univariate regression analyses for the prediction of a complete pathological response by various baseline patient, tumour characteristics and clinical response after two cycles, $2011(\mathrm{~N}=98)$.

\begin{tabular}{|c|c|cc|c|}
\hline \multirow{2}{*}{ Variable } & \multirow{2}{*}{ Value } & \multicolumn{3}{|c|}{ Univariate regression model } \\
\cline { 3 - 5 } & & Odds ratio & P value & $95 \%$ CI \\
\hline Response after two cycles & Response vs. non-response & 22.5 & 0.003 & $2.9-171.6$ \\
\hline Age & $<50$ vs. >50 years & 1.2 & 0.656 & $0.5-3.2$ \\
\hline Histologic grade & $1+2$ vs. 3 & 0.4 & 0.085 & $0.1-1.0$ \\
\hline ER status & Positive vs. negative & 0.4 & 0.04 & $0.1-1.0$ \\
\hline
\end{tabular}

Table 3: Showing correlation between the histological type of tumors and clinical response, 2011 $(\mathrm{N}=98)$.

\begin{tabular}{|c|c|c|c|c|c|c|}
\hline & \multicolumn{4}{|c|}{ Clinical response } & \multirow[t]{2}{*}{ Tota } \\
\hline & & complete & partial & Stable disease & progressive disease & \\
\hline \multirow{4}{*}{$\begin{array}{l}\text { Histological } \\
\text { type }\end{array}$} & Ductal & 10 & 62 & 4 & 0 & 76 \\
\hline & Lobular & 1 & 2 & 9 & 3 & 15 \\
\hline & Medullary & 0 & 5 & 0 & 0 & 5 \\
\hline & Tubular & 0 & 2 & 0 & 0 & 2 \\
\hline \multicolumn{2}{|l|}{ Total } & 11 & 71 & 13 & 3 & 98 \\
\hline
\end{tabular}

\section{DISCUSSION}

The use of neoadjuvant chemotherapy to treat locally advanced breast cancer has been shown to be efficacious. In the National Surgical Adjuvant Breast and Bowel Project B-18, an objective response was seen in $80 \%$ of 747 patients after they received neoadjuvant chemotherapy (6). In our study, the overall clinical response rate was $83.6 \%$. This high percentage permits breast conserving surgery in one third of such patients. The short-term effect of neoadjuvant chemotherapy on breast cancer is positive, and clinical response, even pCR, may be attained in some patients (7).

There still might be residual tumor histologically in patients who achieved complete clinical response (8-10). In our study, 11 patients (12\%) completed clinical response, 6 of them completed pathological response, and the other 5 had residual disease histologically.

Several groups have proposed breastsparing radiotherapy for patients who achieved complete clinical response (11). Jacquillat et al. noted an overall breast recurrence rate of $6.0 \%$ among 250 patients treated with neoadjuvant chemotherapy followed by breast radiotherapy
(12). Accurate estimation of the tumor size after neoadjuvant chemotherapy is crucial for deciding the type and extent of operation to be performed.

Assessing residual disease after the administration of chemotherapy is important in helping to select patients for breast conservation surgery (13). Apart from measuring the tumor diameter directly, imaging techniques such as mammography and ultrasonography may provide further information about the tumor size after chemotherapy (14). Whether Magnetic Resonance Imaging (MRI) can provide a better correlation with the pathological size remains uncertain, but early results appear promising. Cross et al. have shown that the reduction in tumor enhancement on an MRI scan correlates with the extent of the disease as seen at the pathological examination (7).

We have also found that higher grade is independently associated with either complete or partial clinical response in the breast. Eighty eight per cent of patients who developed clinical response had poor grade at the time of diagnosis. There exist numerous studies that have shown better responses in rapidly proliferating tumors 
with a higher grade (Petit et al. 2004; Guarneri et al. 2006; Penault et al. 2002) (15-17).

In concordance with previous studies, we have observed a positive correlation with estrogen receptors and overall (clinical and pathological) response. Surprisingly, a higher objective response rate (cCR+ pCR) was seen in patients who are ER-positive as compared with ER-negative patients ( $\mathrm{p}=0.004)$, with $94 \%$ of ER-positive tumors responding, compared to only $57 \%$ of ER negative tumors. $80 \%$ of the patients who achieved cCR are ER-positive. All the patients who attained pCR are ER-positive. This finding seems to contradict the finding from Danishad et al. (2006), who identified that ER negative tumors respond better for chemotherapy (17-18).

A varying correlation between clinical response and histological type of tumors has been reported in previous studies. For example, Brifford et al. reported a highly significant clinical response in patients with invasive ductal carcinoma (IDC)(19). Mathieu et al. and Newman et al. reported that invasive lobular carcinoma (ILC) is an independent predictor of ineligibility for BCS after neoadjuvant chemotherapy compared with IDC (20). Although all these studies show that ILC patients are less likely to achieve BCS after neoadjuvant chemotherapy, they do not address whether the use of neoadjuvant chemotherapy improves the baseline BCS rates for ILC patients (20-21). In our study, seventy-two out of 76 patients diagnosed as invasive ductal carcinoma achieved clinical response (complete or partial) to NAC (Table 3). A complete clinical response was seen in only one patient with ILC (1\%), which is also consistent with other ILC series in the literature (21). No lobular carcinomas had a complete pathological response to NAC in this study. These findings suggest that histological type in breast carcinoma may play an important role in predicting the degree of tissue response and pathologic response to NAC. More importantly, they raise the question as to whether NAC should be used in cases of invasive lobular carcinoma.

In conclusion, there is strong evidence that treatment of locally advanced breast cancer with neoadjuvant chemotherapy will downgrade the tumor, increasing the opportunity for more conservative surgery.

\section{REFERENCES}

1. El-Sayed MI, Maximous DW, Aboziada MA, Abdel-Wanis ME, Mikhail NN. Feasibility of breast conservation after neoadjuvant taxene based chemotherapy in locally advanced breast cancer: a Prospective Phase I trial. Ann Surg Innov Res. 2010;4:5.

2. Mathew J, Asgeirsson KS, Cheung KL, Chan S, Dahda A, Robertson JF. Neoadjuvant chemotherapy for locally advanced breast cancer: a review of the literature and future directions. Eur J Surg Oncol. 2009 Feb; 35(2):113-22.

3. Ustaalioglu BB, Gumus M, Bilici A, Seker M, Dane F, Salepci T, et al. Neoadjuvant chemotherapy for locally advanced breast cancer: a single center experience. Med Oncol. 2010 Jun;27(2):454-8.

4. El Saghir NS, Eniu A, Carlson RW, Aziz Z, Vorobiof D, Hortobagyi GN. Locally advanced breast cancer: treatment guideline implementation with particular attention to low- and middle-income countries. Cancer. 2008 Oct 15;113(8 Suppl):2315-24.

5. Elgaili EM, Abuidris DO, Rahman M, Michalek AM, Mohammed SI. Breast cancer burden in central Sudan. International Journal of Women's Health. 2010; 2:77-82.

6. Buzdar AU. Preoperative chemotherapy treatment of breast cancer: a review. Cancer. 2007 Dec 1;110(11):2394-407.

7. Londero V, Bazzocchi M, Del Frate C, Puglisi F, Di Loreto C, Francescutti G, et al. Locally advanced breast cancer: comparison of mammography, sonography and MR imaging in evaluation of residual disease in women receiving neoadjuvant chemotherapy. Eur Radiol. 2004 Aug;14(8):1371-9.

8. Fernandez-Sanchez M, Gamboa-Dominguez A, Uribe N, Garcia-Ulloa AC, FloresEstrada D, Candelaria M, et al. Clinical and pathological predictors of the response to neoadjuvant anthracycline chemotherapy in locally advanced breast cancer. Med Oncol. 2006;23(2):171-83. 
9. Schwartz G. Neoadjuvant induction chemotherapy. Minerva Ginecol. 2005 Jun;57(3):327-48.

10. Charfare H, Limongelli S, Purushotham AD. Neoadjuvant chemotherapy in breast cancer. Br J Surg. 2005 Jan;92(1):14-23.

11. Clouth B, Chandrasekharan S, Inwang R, Smith S, Davidson N, Sauven P. The surgical management of patients who achieve a complete pathological response after primary chemotherapy for locally advanced breast cancer. Eur J Surg Oncol. 2007 Oct;33(8):961-6.

12. Waljee JF, Newman LA. Neoadjuvant systemic therapy and the surgical management of breast cancer. Surg Clin North Am. 2007 Apr;87(2):399-415, ix.

13. Chong HY, Taib NA, Rampal S, Saad M, Bustam AZ, Yip CH. Treatment options for locally advanced breast cancer--experience in an Asian tertiary hospital. Asian Pac J Cancer Prev. 2010;11(4):913-7.

14. Jorgensen J, Cold S, Kamby C. [Primary inoperable breast cancer]. Ugeskr Laeger. 2007 Sep 10;169(37):3091-3.

15. Gogas H, Pectasides D, Kostopoulos I, Lianos E, Skarlos D, Papaxoinis G, et al. Paclitaxel and carboplatin as neoadjuvant chemotherapy in patients with locally advanced breast cancer: A phase II Trial of the Hellenic Cooperative Oncology Group. Clin Breast Cancer. 2010 Jun; 10(3):230-7.

16. Costa SD, Loibl S, Kaufmann M, Zahm DM, Hilfrich J, Huober J, et al. Neoadjuvant chemotherapy shows similar response in patients with inflammatory or locally advanced breast cancer when compared with operable breast cancer: a secondary analysis of the GeparTrio trial data. J Clin Oncol. 2010 Jan 1;28(1):83-91.

17. Alvarado-Cabrero I, Alderete-Vazquez G, Quintal-Ramirez M, Patino M, Ruiz E. Incidence of pathologic complete response in women treated with preoperative chemotherapy for locally advanced breast cancer: correlation of histology, hormone receptor status, Her2/Neu, and gross pathologic findings. Ann Diagn Pathol. 2009 Jun;13(3):151-7.

18. Newman LA. Management of patients with locally advanced breast cancer. Curr Oncol Rep. 2004 Jan;6(1):53-61.

19. Beresford MJ, Stott D, Makris A. Assessment of clinical response after two cycles of primary chemotherapy in breast cancer. Breast Cancer Res Treat. 2008; 109:5.

20. Boughey JC, Wagner J, Garrett BJ. Neoadjuvant Chemotherapy in Invasive Lobular Carcinoma May Not Improve Rates of Breast Conservation. Ann Surg Oncol. [ORIGINAL]. 2009; 16:5.

21. Sullivan PS, Apple SK. Should histologic type be taken into account when considering neoadjuvant chemotherapy in breast carcinoma? Breast J. 2009 Mar-Apr;15 (2):146-54. 